\title{
Asymmetric Circular Array Antenna Synthesis Based on Angular Positions
}

\author{
Mangolika Bhattacharya, Sudipta Das, Durbadal Mandal, and Anup Kumar Bhattacharjee
}

\begin{abstract}
In this paper, a broadside circular array antenna resting on $x-y$ plane is assumed. Design aim here is to obtain the optimal combination of non-uniform angular positions of the elements and uniform radius for the array, so as to acquire the radiation with the lowest possible sidelobe peak (SLL) with least possible first null beamwidth (BWFN) increment. Optimum angular locations of elements are found using Real coded Genetic Algorithm (RGA).
\end{abstract}

Index Terms-Circular array, asymmetric circular array, sidelobe reduction, optimal angluar location, real coded genetic algorithm.

\section{INTRODUCTION}

A mass of research operations have been conducted in the previous few decades on various antenna array structures in order to acquire shrunken relative peak sidelobe level and the first null beamwidth [1]-[28]. Antenna Array is constituted by congregation of radiating elements in an electrical or geometrical conformation. Entire field of the Antenna Array is calculated by vector summation of the fields radiated by each single element. A circular array has all its elements placed along a perimeter of a circle. Circular array is a layout that has a contour of huge practical involvement. Its application span extends in radio detection finding, air and space navigation, underground propagation, radar sonar and many other systems. The aim of antenna array geometry synthesis is to assess the physical setup of the array that produces the radiation pattern that is nearest to the desired pattern [29]. Various kinds of works have been carried out to modify the radiation pattern of basic and concentric circular arrays by optimizing its geometry. Major aspects of optimizing circular geometry include sidelobe performance improvement [2], [17], [18], [22], [28]; adaptive-beamforming [13]; mutual coupling [21]; thinning [25] etc. In this paper the design aim is to inhibit the proportion of the maximum sidelobe level (SLL) to main beam level for a circular antenna array. Besides suppressing maximum relative sidelobe level the goal is to inflict nulls for each and every peak of sidelobe and simultaneously conserving the nulls of the initial pattern at their locations as well as to restrain the dispersion of main beam. In the present

Manuscript received February 25, 2012; revised April 26, 2012.

M. Bhattacharya is with the Aryabhatta Institute of Engineering and Management, Durgapur, West Bengal, India (e-mail: mangolika@gmail.com).

S. Das, D. Mandal, A. Kumar Bhattacharjee are associated with the Department of Electronics and Communication Engineering, National Institute of Technology Durgapur, Durgapur, and West Bengal, India. (email sudipta.sit59@gmail.com,durbadal.bittu@gmail.com,akbece12@yahoo.co $\mathrm{m})$. case, the angular positions of elements and the radius of the array are chosen as the parameter for qualifying the radiation pattern. A radiation pattern with lowest SLL, more and deeper nulls, and, slenderer main beam is advantageous in the present case. RGA [30] is employed in this paper to obtain the desired pattern [7], [26], [30].

Remaining of the paper is formatted as follows: in section II, the general design equations for the non-uniformly placed asymmetric circular antenna array are stated. Then, in section III, RGA is presented in brief. Numerical simulation outcomes are demonstrated in section IV. Finally the paper concludes with a summary of the work in section V.

\section{Design EQuation}

Figs. 1 and 2 depict the geometry of a circular array of $\mathrm{N}$ isotropic sources is laid on $\mathrm{x}-\mathrm{y}$ plane having a radius a and scanning at point $\mathrm{P}$ in the far field. Array factor $(A F(\theta, \phi))$ of such an array can be given by (1) [30].

$$
A F(\theta, \phi)=\sum_{n=1}^{N} I_{n} e^{\left[j k a \sin \theta \cos \left(\phi-\phi_{n}\right)+\alpha_{n}\right]}
$$

where,

$I_{n}=$ individual excitation amplitude,

$k=2 \pi / \lambda, \lambda$ being the wavelength of operation,

$\theta$ is the azimuth angle,

$\phi$ is the elevation angle,

$\phi_{n}$ is the angular location of $\mathrm{n}^{\text {th }}$ element along $x-y$ plane.

$\alpha_{n}=-k a \sin \theta_{0} \cos \left(\phi_{0}-\phi_{n}\right)$

$\left(\theta_{0}, \phi_{0}\right)$ is the desired direction of scanning. Therefore there should be maxima of $A F(\theta, \phi)$ at that point. In the present case, the maximum scanning is required along the $z$ axis. Therefore, $\theta_{0}=0$ for this case. Moreover, in the paper, the pattern in the $x-z$ plane is taken for experiment. So, $\phi=0$ in the present case. Thus, (1) changes to,

$$
A F\left(\theta, \phi_{n}\right)=\sum_{n=1}^{N} I_{n} e^{j k a \sin \theta \cos \phi_{n}}
$$

For initial case, $\phi_{n}$ was equal to $2 \pi n / N$ i.e. all the elements were uniformly spaced. Initial radius for each structure is taken as $3 \lambda /\{8 \sin (\pi / N)\}$.

The design goal in the present case is to find the optimum set of values of $\phi_{n}$ in order to get the minimum SLL for least BWFN increment. Element positions along the perimeter are varied asymmetrically and simultaneously a probable optimal radius is found in order to get the desired pattern in the 
present case. A radiation pattern that gives lowest possible SLL and thin BWFN is preferred for this case.

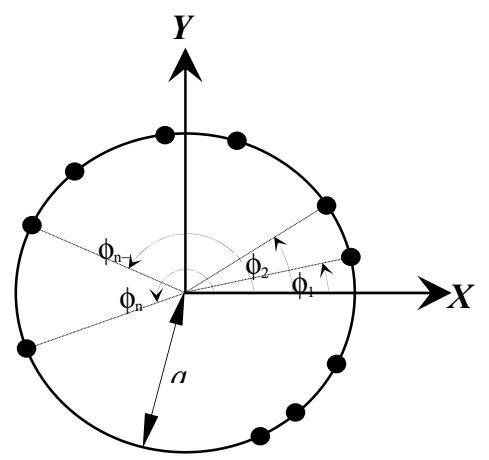

Fig. 1. Geometry of $N$ element Asymmetric circular array of isotropic radiators (Top View)

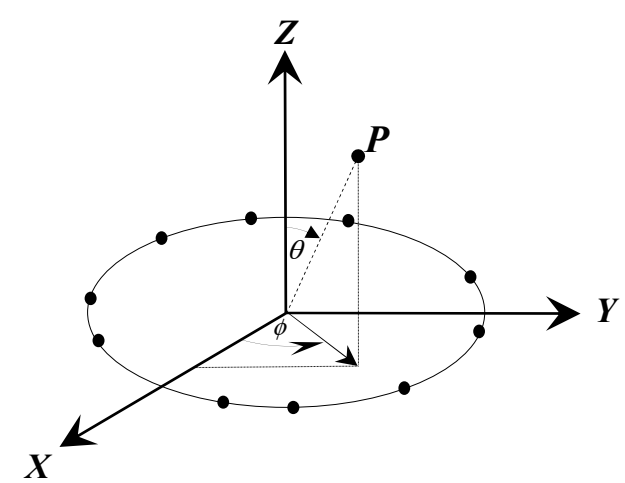

Fig. 2. An Asymmetric circular array laid in the $x-y$ plane with $N$ isotropic elements scanning at a point $P$ in the far field

The Cost Function is designed to make the problem a minimization problem. It is designed in such a way that, reduction of SLL in both the upper and the lower bands without significant increment in the BWFN causes lowering of cost function. In the present case the fitness function or the cost function " $C F$ " is taken as below,

$$
\begin{aligned}
C F & =\frac{S L L_{\text {initial }}}{S L L_{\text {current }}}+\left|A F\left(\theta_{i}, \phi_{n}\right)\right|^{2} \\
& +\left|B W F N_{\text {initial }}-B W F N_{\text {current }}\right|
\end{aligned}
$$

where,

$$
\begin{aligned}
& S L L_{\text {initial }}=20 \log _{10}\left(\frac{\left.0.5 \times \mid \begin{array}{c}
A F\left(\theta_{\text {msl } 1_{\text {mintal }}}, I_{\text {initial }}\right) \\
+A F\left(\theta_{\text {msl }} 2_{\text {mital }}, I_{\text {initial }}\right.
\end{array}\right)}{\mid A F\left(\theta_{0}, I_{\text {initial }} \mid\right.}\right)
\end{aligned}
$$

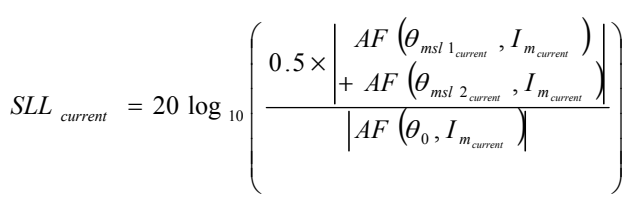

In the present case, $\theta_{0}$ is the angle where peak of the main lobe is attained in $\theta \in\left(-\frac{\pi}{2}, \frac{\pi}{2}\right) . \theta_{m s l_{\text {current }}}$ is the angle where the maximum sidelobe $\left(A F\left(\theta_{m s 1_{1_{\text {current }}}}, I_{m_{\text {current }}}\right)\right)$ is attained in the lower band and $\theta_{m s l_{\text {current }}}$ is the angle where the maximum sidelobe $\left(A F\left(\theta_{m s l_{1_{\text {current }}}}, I_{m_{\text {current }}}\right)\right)$ is attained in the upper band for the current iteration. Similarly, $\theta_{m s l l_{\text {initial }}}$ is the angle where the maximum sidelobe $\left(A F\left(\theta_{m s l_{1 \text { initial }}}, I_{\text {initial }}\right)\right)$ is attained in the lower band and $\theta_{m s l_{\text {initial }}}$ is the angle where the maximum sidelobe $\left(A F\left(\theta_{m s l_{\text {intital }}}, I_{\text {initial }}\right)\right)$ is attained in the upper band for the initial case. If the currecnt iteration has the lower SLL, the 1st term will get reduced and hence cost function will be reduced. So, the first term in (3) is used for minimizing the SLL.

The second term in (3) is used [16] to introduce nulls in each and every direction outside the main beam. Thus this term, in the present case is used to reduce sidelobe level in each iteration.

In (3) the two beamwidths, $B W F N_{\text {current }}$ and $B W F N_{\text {initial }}$ basically refer to the computed first null beamwidths in radian for the non-uniform angular positions case and for uniform angular positions (initial case) respectively. So, the third term of (3) [25], [26] restricts the spreading of the main beam as far as possible. The Real coded Genetic Algorithm (RGA) employed for optimizing the angular locations of the elements, resulting in the minimization of $C F$.

\section{REAl CODED Genetic Algorithm (RGA)}

GA is mainly a probabilistic seeking technique, establised on the rules of natural selection and evolution [27]. At each generation it conserves a collection of adoptees where each adoptee is a encrypted kind of a possible result of the problem at hand and called chromosome. Chromosomes are created with genes of arbitrary values between $(0,1)$. Each chromosome is valuated by a function known as fitness function, which is generally the cost function or the objective function of the matching optimization problem. In our case the cost function " $C F$ " is given by (3).

Steps of RGA as applied for optimization of Angular Locations of Elements for Asymmetric Circular Array Antennas are [7]:

- Initialization of real chromosome strings of $n_{\mathrm{p}}$ population, each constituting of a set of Angular Locations of Elements. Size of the set counts on the number of antenna elements in a specific array design.

- Decipherment of strings and evaluation of $C F$ of each string.

- Extraction of elite strings in order of increasing $C F$ values from the minimum value.

- Copying of the elite strings over the non-selected strings.

- Crossover and mutation to generate off-springs.

- Genetic cycle updating.

The iteration stops when the maximum number of cycles is reached. The grand minimum and its matching chromosome string or the desired solution are finally obtained.

\section{RESUlts OF SimUlation}

This section gives the simulated results for various linear antenna array designs optimized by RGA technique. Three 
sets of Circular array structures having 16, 18, and 20 elements are considered, each having uniform excitation coefficients of the elements.

The parameters for the RGA are set after many trial runs. It is found that the best results are obtained for an initial population of 120 chromosomes. Maximum number of Generations $N_{m}$ is limited to 250 . For selection operation, the method of natural selection is chosen with a selection probability of 0.3 . Crossover is randomly selected dual points. Crossover ratio is 0.8 . Mutation probability is 0.04 .

Table I shows the maximum sidelobe level and the BWFN for all the sets of asymmetric uniform circular array antenna designs having the initial uniform angular spacing variable as $\phi_{n}=2 n \pi / N$.

Table II gives the optimal individual non-uniform angular spacing varied asymmetrically in the range $\left(0^{\circ}, 360^{\circ}\right)$ with optimal radius. Optimal non-uniform angular locations along with optimal radius for 16, 18 and 20 element asymmetric circular array are tabulated here. These values corresponding to each set are calculated using (2) and the fitness function as described in (3). In this table results are found for $\phi_{n} \in(0,2 \pi)$. In both the tables SLL and BWFN are expressed in $\mathrm{dB}$ and degree respectively. In Table II optimal angular locations are also expressed in degree.

\section{A. Analysis of Radiatin Pattern of Asymmetric Non-Uniform Circular Array Sets}

Figs. 3-4 examine and compare the initial radiation pattern of the 18 and 20 elements asymmetric uniform circular antenna array with corresponding asymmetric circular antenna array with optimum non-uniform angular locations of elements respectively.

TABLE I: MAXIMUM SIDELOBE LEVEL (SLL) AND FIRST NULL BEAMWIDTH (BWFN) FOR DIFFERENT SETS OF ASYMMETRIC CIRCULAR ARRAY ANTENNA WITH UNIFORM EXCITATNON AND UNIFORM ANGULAR

\begin{tabular}{llc}
\multicolumn{3}{c}{ SPACING } \\
\hline \hline No. of Elements (El.) & Initial max SLL $(\mathrm{dB})$ & Initial BWFN $\left(^{\circ}\right)$ \\
\hline 16 & -7.90 & 23.00 \\
18 & -7.90 & 20.50 \\
20 & -7.90 & 18.30 \\
\hline \hline
\end{tabular}

TABLE II: MAXIMUM SIDELOBE LEVEL (SLL) AND FIRST NULL BEAMWIDTH (BWFN) FOR DIFFERENT SETS OF ASYMMETRIC CIRCULAR ARRAY ANTENNA WITH UNIFORM EXCITATNON AND UNIFORM ANGULAR SPACING

\begin{tabular}{|c|c|c|c|c|c|c|}
\hline El & \multicolumn{3}{|c|}{$\begin{array}{l}\text { Optimal Angular Locations } \\
\text { of elements froom positive } \\
x \text {-axis }\left({ }^{\circ}\right)\end{array}$} & \multirow[t]{2}{*}{$\begin{array}{l}\text { Optimal } \\
\text { Radius } \\
(\lambda)\end{array}$} & \multirow[t]{2}{*}{$\begin{array}{l}\text { Final } \\
\text { SLL } \\
(\mathrm{dB})\end{array}$} & $\begin{array}{c}\text { Final } \\
\text { BWFN } \\
\left({ }^{\circ}\right)\end{array}$ \\
\hline \multirow{5}{*}{16} & 5.0753 & .3956 .7885 & 0297.57 & & & \multirow{5}{*}{33.60} \\
\hline & 102.76 & 227.34 & 239.37 & \multirow{4}{*}{2.46} & \multirow{4}{*}{-18.43} & \\
\hline & 251.29 & 256.00 & 274.87 & & & \\
\hline & 277.05 & 282.50 & 288.26 & & & \\
\hline & 299.08 & 326.14 & & & & \\
\hline \multirow{6}{*}{18} & 0.67 & $45.04 \quad 62$. & 275.64 & \multirow{6}{*}{2.16} & \multirow{6}{*}{-21.38} & \multirow{6}{*}{31.80} \\
\hline & 87.19 & $94.91 \quad 96.37$ & 105.57 & & & \\
\hline & 129.59 & 164.28 & 230.46 & & & \\
\hline & 245.62 & 252.48 & 277.98 & & & \\
\hline & 283.69 & 298.46 & 309.33 & & & \\
\hline & 331.77 & & & & & \\
\hline \multirow{6}{*}{20} & 0.4243 & .2054 .5260 & .753 .49 & \multirow{6}{*}{2.40} & \multirow{6}{*}{-19.64} & \multirow{6}{*}{27.20} \\
\hline & 87.63 & 99.66 & 08.0423 & & & \\
\hline & 111.68 & 121.31 & 135.06 & & & \\
\hline & 145.06 & 184.53 & 235.03 & & & \\
\hline & 258.02 & 268.87 & 274.77 & & & \\
\hline & 281.76 & 297.31 & 322.33 & & & \\
\hline
\end{tabular}

These figures are framed straightaway from the values of corresponding sets of Tables I and II respectively. From the figures it is illustrated that optimizing with (3) enriches the radiation pattern by decreasing the SLL.

It can be viewed from the figures and tables that SLL reduction has been attained at the cost of BWFN. As seen from the Table II, an asymmetric circular array antenna with optimal non-uniform angular locations of elements has SLL reduced to $-21.317 \mathrm{~dB}$ and $-19.643 \mathrm{~dB}$ against $-7.90 \mathrm{~dB}$ and $-7.90 \mathrm{~dB}$ for the set having 18 and 20 elements respectively.

\section{B. Convergence Profile of $R G A$}

The minimum $C F$ values against number of iteration cycles are registered to obtain the convergence profile of each set. Figs. 5 and 6 displays the convergence profiles of minimum $C F$ of non-uniform asymmetric circular antenna array set having 18 and 20 elements respectively. It can be viewed that the convergence curve of an 18 element circular antenna array converges after 150 iterations, and, the convergence curve for 20 element array converges after 180 iterations. The programming has been written in MATLAB language using MATLAB 7.5 on core (TM) 2 duo processor, $1.83 \mathrm{GHz}$ with $2 \mathrm{~GB}$ RAM.

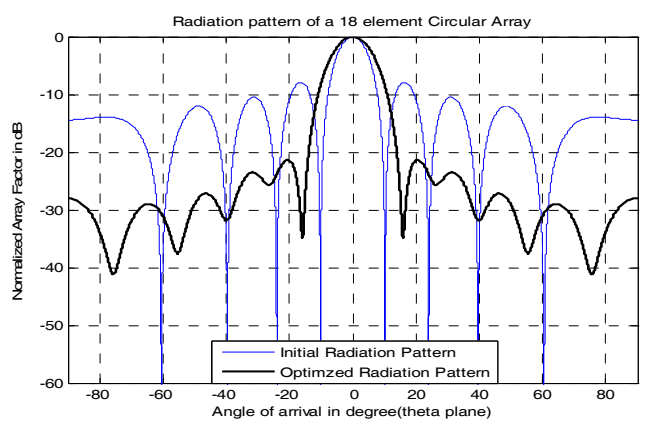

Fig. 3. X-z plane Radiation pattern of a 18 element asymmetric circular array on the $\mathrm{x}-\mathrm{y}$ plane

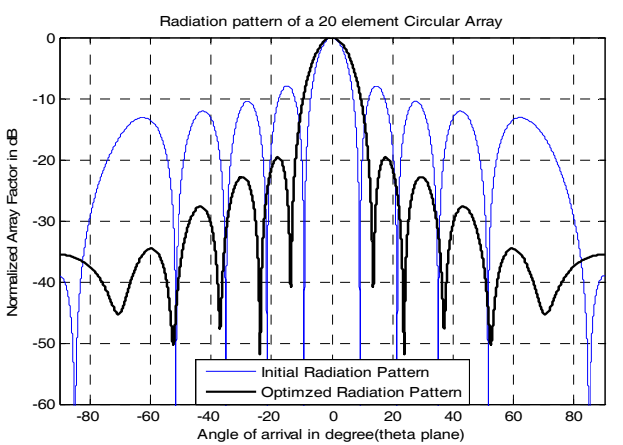

Fig. 4. $x-z$ plane Radiation pattern of a 20 element asymmetric circular array on the $x-y$ plane

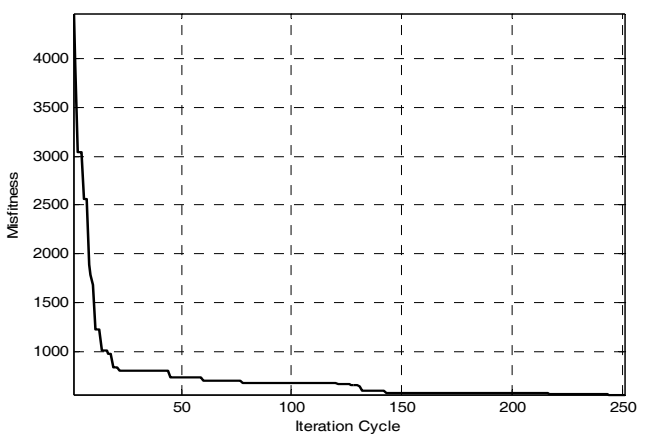

Fig. 5. Convergence profile for a 18 element non-uniform asymmetric circular array antenna using RGA. 


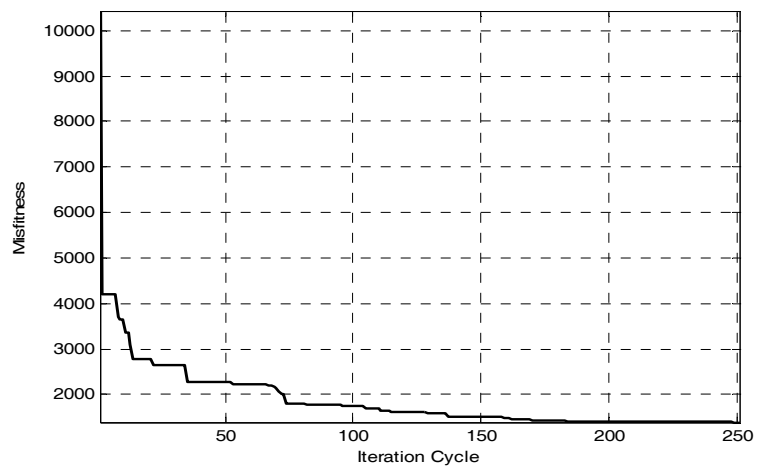

Fig. 6. Convergence profile for a 20 element non-uniform asymmetric circular array antenna using RGA.

\section{CONCLUSION}

In this paper, the design of an asymmetric circular array antenna with non-uniform angular spacing between the elements has been depicted applying the proficiency of RGA. Simulated results reveal that optimizing the radiation pattern by changing the references asymmetrically causes vanishing of deep Nulls. Again, varying the same non-uniformly provides an appreciable SLL reduction and more Nulls with respect to corresponding circular array with consistent angular spacing amongst elements.

The asymmetric linear antenna array sets having 18 and 20 elements optimized using cost function as in (3) have the final SLL reduced to $-21.38 \mathrm{~dB}$ and $-19.64 \mathrm{~dB}$ against -7.90 $\mathrm{dB}$ of initial value respectively. The results for these sets show that the radius tends to take its initial value. Again, the corresponding optimized radiation pattern has some extra nulls.

Hence, with the help of RGA a circular array antenna with lower interference from the unwanted directions is reconstructed with slight sacrifice in directivity. Thus, the RGA technique is anticipated to be a very bright evolutionary optimization technique for the global optimization of any antenna array design problem.

\section{REFERENCES}

[1] K. Murthy and A. Kumar, "Synthesis of Linear Antenna Arrays," IEEE Trans. Antennas Propagat. pp. 865-870, 1976.

[2] F. Hodjat and S. A. Hovanessian, "Non- uniformly spaced linear and planar array antennas for sidelobe reduction," IEEE Trans. on Antennas and Propagation, No. 2, pp. 198-204, 1978.

[3] B. P. Ng, M. H. Er, and C. A. Kot, "Linear array aperture synthesis with minimum sidelobe level and null control," Inst. Elect. Eng. Proc. Microw. Antennas Propagat. vol. 141, no. 3, 1994.

[4] C. C. Yu, "Sidelobe reduction of asymmetric linear array by spacing perturbation," Electronics Letters, vol. 33, no. 9, 1997.

[5] K. K. Yan and Y. Lu, "Sidelobe Reduction in Array-Pattern Synthesis Using Genetic Algorithm," IEEE Trans. Antennas Propagat, vol. 45, no. 7, pp. 1117-1122, 1997

[6] K. K. Yan and Y. Lu, "Sidelobe Reduction in Array-Pattern Synthesis Using Genetic Algorithm," IEEE Trans. Antennas Propagat. vol. 45, no. 7, 1997.

[7] F. J. Ares-Pena, J. A. Rodriguez-Gonzalez, E. Villanueva-Lopez and S R. Rengarajan, "Genetic Algorithms in the Design and Optimization of Antenna Array Patterns," IEEE Trans. Antennas Propagat, vol. 47, no. 3, 1999.

[8] B. P. Kumar and G. R. Branner, "Design of Unequally Spaced Arrays for Performance Improvement," IEEE Trans. Antennas Propagat, vol. 47, no. 3, 1999.
[9] D. Marcano and F. Duran, "Synthesis of Antenna Arrays Using Genetic Algorithms," IEEE Antennas Propagat. Magazine, vol. 42, no. 3, 2000.

[10] A. Lommi, A. Massa, E. Storti, and A. Trucco, "Sidelobe reduction in sparse linear arrays by genetic algorithms," Microwave and Optical Technology Letter, vol. 31, no. 3, pp. 194-196, 2002.

[11] D. G. Kurup, M. Himdi, and A. Rydberg, "Synthesis of uniform amplitude unequally spaced antenna arrays using the differential evolution algorithm," IEEE Trans. Antennas Propagat, vol. 51, no. 9, pp. 2210-2217, 2003.

[12] L. L. Wang and D. G. Fang, "Synthesis of nonuniformly spaced arrays using genetic algorithm," In Proc. Asia-Pacific Conference on Environmental Electromagnetics, pp. 302-305, 2003.

[13] Li, Y, K. C. Ho, and C. Kwan, "A novel partial adaptive broad-band beamformer using concentric ring array," in Proc. IEEE ICASSP'04, II-177-II-180, Montreal, Quebec, Canada, 2004.

[14] S. Kyle Smith, J. C. Bregains, K. L. Melde, and F. Ares, "Analytical and Optimization Methods For Linear Arrays with High efficiency and Low Side lobes," in Proc. IEEE AP-S Int. Symp., vol. 1, pp. 547-550, 2004.

[15] B. P. Kumar and G. R. Branner, "Generalized analytical technique for the synthesis of unequally spaced arrays with linear, planar, cylindrical or spherical geometry," IEEE Trans. Antennas Propag, vol. 53, no. 2, pp. 621-633, 2005.

[16] M. M. Khodier and C. G. Christodoulou, "Linear Array Geometry Synthesis With Minimum Sidelobe Level and Null Control Using Particle Swarm Optimization," IEEE Trans. Antennas Propagat, vol. 53, no. 8, pp. 2674-2679, 2005.

[17] M. Dessouky, H. Sharshar, and Y. Albagory, "Efficient sidelobe reduction technique for small-sized concentric circular arrays," Progress In Electromagnetics Research, vol. PIER 65, pp. 187-200, 2006.

[18] S. M. A. Panduro, A. L. Mendez, R. Dominguez, and G. Romero, "Design of non-uniform circular antenna arrays for side lobe reduction using the method of genetic algorithms," Int. J. Electron.Commun. (AEÜ) vol. 60, pp. $713-717,2006$.

[19] S. H. Son and U. H. Park, "Sidelobe reduction of Low-Profile Array Antenna Using a Genetic Algorithm," ETRI Journal, Vol. 29, no. 1, 2007.

[20] P. J. Bevelacqua and C. A. Balanis, "Optimizing antenna array geometry for interference suppression," IEEE Trans. Antennas Propag., vol. 55, no. 3, pp. 637-641, 2007.

[21] R. Fallahi and M. Roshandel, "Effect of mutual coupling and configuration of concentric circular array antenna on the signal-tointerference performance in CDMA systems," Progress In Electromagnetic Research, vol. 76, pp. 427-447, 2007.

[22] R. L. Haupt, "Optimized element spacing for low sidelobe concentricring arrays," IEEE Trans. Antennas Propag. vol. 56, pp. 266-268,Jan, 2008.

[23] N. Pathak, G. K. Mahanti, S. K. Singh, J. K. Mishra and A. Chakraborty, "Synthesis of Thinned Planar Circular Array Antennas Using Modified Particle Swarm Optimization," Progress In Electromagnetic Research Letters, vol. 12, pp. 87-97, 2009.

[24] W. C. Barott and P. G. Steffes, "Grating Lobe Reduction in Aperiodic Linear Arrays of Physically Large Antennas," IEEE Antennas and Wireless Propagation Letters, vol. 8, 2009.

[25] D. Mandal, S. K. Ghoshal, S. Das, S .Bhattacharjee, and A. K. Bhattacharjee, "Improvement of Radiation Pattern for Linear Antenna Array Using Genetic Algorithm," in Proc. IEEE International Conference on Recent Trends in Information, Telecommunication and Computing (ITC 2010), pp. 126-129, 2010.

[26] S. Das, S. Bhattacharjee and D. Mandal, "Improvement of Far Field Radiation Pattern of Linear Array Antenna Using Genetic Algorithm," ICTACT Journal of Communication Technology, pp. 23-32, 2010.

[27] S. Das, S. Bhattacharjee, A. K. Bhattacharjee, and D. Mandal "Sidelobe Level and Null Control of Asymmetric Linear Antenna Array using Genetic Algorithm," International Journal of Artificial Intelligence and Computational Research, vol. 2, no. 1, pp. 7-12, 2010.

[28] D. Mandal, S. P. Ghoshal, and A. K. Bhattacharjee, "Radiation Pattern Optimization for Concentric Circular Antenna Array With Central Element Feeding Using Craziness Based Particle Swarm Optimization," International Journal of $R F$ and Microwave Computer-Aided Engineering, vol. 20, pp. 577-586, 2010.

[29] C. A. Ballanis, Antenna Theory Analysis and Design, 2nd edition, John Willey and Son's Inc, New York, 1997, ch. 6, pp. 324-328.

[30] R. L. Haupt, and D. H. Werner, Genetic Algorithms in Electromagnetics, IEEE Press Wiley-Interscience, 2007. 


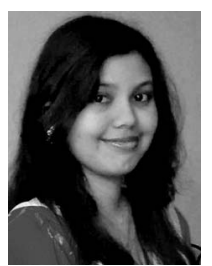

Mangolika Bhattacharya was born in Durgapur, West Bengal, $2^{\text {nd }}$ January 1990 . She is currently a undergraduate student in West Bengal University of Technology pursuing her Final semester of B-Tech degree in Electrical \& Electronics Engineering. She has 1 international journal and 1 international conference paper. Her areas of interest include Evolutionary Optimization Techniques, Array Antenna and Computational Electromagnetics

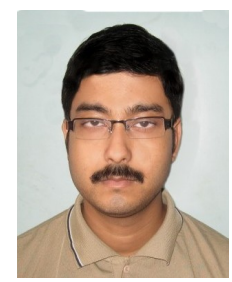

Sudipta Das was born in Malda town of West Bengal, India. on 28th January, 1986. He received B. Tech degree in Electronics and Communication Engineering from the West Bengal University of Technology in 2007 and received his M.Tech degree in Telecommunication engineering in 2010 from National Institute of Technology, Durgapur, India $\mathrm{He}$ is currently working towards his $\mathrm{Ph}$. D. in the department of Electronics and Communication Engineering from National Institute of Technology, Durgapur. He has more than 16 papers published in the international jouornals and conferences.

He became member of IEEE in the year 2010. His research interests are in the areas of Electromagnetics, Microwave Circuits, and ,Designing Antennas and Antenna Arrays and Evolutionary Algorithms.

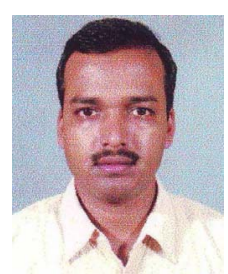

Durbadal Mandal was born in Bankura district, West Bengal in India in the year 1973. He received his B. E. degree in Electronics and Communication Engineering, from Regional Engineering College, Durgapur, West Bengal, India in the year 1996 and received his M.Tech degree in Electronics and Communication Engineering with specialization in Telecommunication Engineering from National Institute of Technology, Durgapur, West Bengal, India in the year 2008 and received Ph.D. degree from National Institute of Technology, Durgapur, in the year 2011. He is currently working as Assistant Professor of Electronics and Communication Engineering at National Institute of Technology, Durgapur, India. He has more than 98 papers published in national and international journals and conferences.

He became member of IEEE in the year 2010. His research interests include Evolutionary Optimization Techniques, Array Antenna Optimization and Digital Filter Optimization

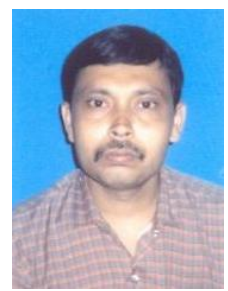

A. K. Bhattacharjee was born in Malda of West Bengal, India, on $19^{\text {th }}$ January 1962 . He received his B. E. degree in electronics and telecommunications engineering, from BE College, Shibpur, West Bengal, India in the year 1983. He received the M. E. and $\mathrm{Ph}$. D. degrees from Jadavpur University, Kolkata, West Bengal, India in the year 1985 and 1989 respectively. Presently, he is attached with National Institute of Technology, Durgapur, West Bengal, India, as Professor in the Department of electronics and communication engineering. He has published more than 133 papers in national and international conferences and journals

His basic research work is in the areas of Microstrip Antenna, Cryptography and Array Antenna Optimization. 